\title{
10058-F4, a c-Myc inhibitor, markedly increases valproic acid-induced cell death in Jurkat and CCRF-CEM T-lymphoblastic leukemia cells
}

\author{
QITIAN MU ${ }^{1,2,3}$, QIULING MA ${ }^{1,2}$, SHASHA LU ${ }^{1,2}$, TING ZHANG ${ }^{1,2}$, MENGXIA YU $^{1,2}$, \\ XIN HUANG ${ }^{1,2}$, JIAN CHEN ${ }^{1,2}$ and JIE JIN ${ }^{1,2}$
}

${ }^{1}$ Department of Hematology, Institute of Hematology, The First Affiliated Hospital, Zhejiang University School of Medicine;

${ }^{2}$ Key Laboratory of Hematopoietic Malignancies of Zhejiang Province, Hangzhou, Zhejiang 310003;

${ }^{3}$ Laboratory of Stem Cell Transplantation, Ningbo First Hospital, Ningbo, Zhejiang 315010, P.R. China

Received December 12, 2013; Accepted May 29, 2014

DOI: $10.3892 / \mathrm{ol} .2014 .2277$

\begin{abstract}
Adult T-cell acute lymphoblastic leukemia (T-ALL) has a poor prognosis. Although it has been found that activation of Notch1 signaling occurs in $>50 \%$ T-ALL patients, $\gamma$-secretase inhibitors that target Notch1 signaling are of limited efficacy. However, c-Myc is an important direct target of Notch1 and, thus, c-Myc is another potential therapeutic target for T-ALL. Valproic acid (VPA), a histone deacetylase inhibitor, has been reported to treat various hematological malignancies. In the present study, we showed that c-Myc expression, at a transcriptional level, was dose-dependently downregulated in VPA-induced growth inhibition in T-ALL cell lines, Jurkat and CCRF-CEM cells. 10058-F4, a small molecule c-Myc inhibitor, could increase the downregulation of c-Myc and markedly increase the growth inhibition and cell death induced by VPA in Jurkat and CCRF-CEM cells, which was accompanied by obvious cleavage of capase-3. Z-VAD-FMK, a caspase inhibitor, partially prevented the anti-leukemic effect. The results of the present study suggest that c-Myc inhibitors increase cell death induced by VPA in a caspase-dependent and -independent manner, and their combination could be a potent therapeutic strategy for adult T-ALL patients.
\end{abstract}

\section{Introduction}

T-cell acute lymphoblastic leukemia (T-ALL), an aggressive malignancy arising from T-cell progenitors, accounts for $\sim 15 \%$ of ALL cases in children and $\sim 25 \%$ in adults. The

Correspondence to: Professor Jie Jin, Department of Hematology, Institute of Hematology, The First Affiliated Hospital, Zhejiang University School of Medicine, 79 Qingchun Road, Hangzhou, Zhejiang 310003, P.R. China

E-mail: zjuhematolgy@163.com

Key words: Jurkat, CCRF-CEM, valproic acid, c-Myc inhibitors, T-cell acute lymphoblastic leukemia, cell death long-term survival rate for children and adolescents with this disease is $70-75 \%$, while for adults the rate is only $35-40 \%$ in Western populations (1). Over $50 \%$ of patients with T-ALL harbor activation mutations of Notch1 (2), thus, $\gamma$-secretase inhibitors, which block Notch signaling, were a promising target for therapy. However, associated problems, such as limited therapeutic benefit and intestinal toxicity have since been reported (3). Therefore, there is a requirement for the development of novel and effective treatment strategies for adult T-ALL.

Notch1 mutations mainly involve the heterodimerization domain (HD) and the proline, glutamic acid, serine, threonine-rich domain (PEST) (2). The HD mutation results in ligand-independent proteolytic cleavage of Notch1, while the PEST mutation blocks Fbw7 interaction with Notch1 and, thereby, prevents its polyubiquitination and degradation $(3,4)$. The mutations of these two domains result in constitutive activation of the Notch signaling pathway (1). Moreover, 30\% of T-ALL patients harbor inactivating mutations of the Fbw7 gene, which also activate Notch1 signaling (1). It has been demonstrated that c-Myc is a direct and important target gene of Notch1, and its expression levels have been observed to increase along with activation of Notch1 signaling in T-ALL (5). In addition, a previous study showed that the half-life of c-Myc in B-cell or T-cell ALL was markedly prolonged (6). Therefore, c-Myc may be a target for therapy in T-ALL.

Valproic acid (VPA), a histone deacetylase inhibitor (HDACI), has been reported to treat various hematological malignancies (7-9). Previous studies revealed that VPA was more efficacious in combination with other agents, such as idarubicin, 5-azacitidina and all-trans retinoic acid (10-12). In acute myeloid leukemia (AML), VPA inhibited cell growth, mainly by downregulation of c-Myc expression (13). However, the role of c-Myc in the growth inhibition of T-ALL cells induced by VPA remains unclear. We hypothesized that its role in T-ALL was the same as that in AML, and that a c-Myc inhibitor would be able to augment the anti-leukemic effect of VPA. In the present study, the effect of VPA combined with a c-Myc inhibitor (10058-F4) on T-ALL cell lines (Jurkat and CCRF-CEM cells) was investigated. 


\section{Materials and methods}

Cell lines and reagents. T-ALL cell lines, Jurkat and CCRF-CEM cells, were purchased from the American Type Culture Collection (Manassas, VA, USA). These cells were maintained in RPMI-1640 (Gibco-BRL, Grand Island, NY, USA) supplemented with heat-inactivated fetal bovine serum (Gibco-BRL) at $37^{\circ} \mathrm{C}$ in a $5 \% \mathrm{CO}_{2}$ humidified incubator. VPA and 10058-F4 (Sigma-Aldrich, St. Louis, MO, USA) were dissolved in dimethylsulfoxide (DMSO) at $1 \mathrm{M}$ and $20 \mathrm{mM}$ and then stored at $-20^{\circ} \mathrm{C}$ in small aliquots. Z-VAD-FMK (Sigma-Aldrich), a pan-caspase inhibitor (14), was also dissolved in DMSO and its final concentration was $20 \mu \mathrm{M}$.

Cell viability assay. Cell viability was measured by 3-(4,5-dimethylthiazol-2-yl)-2,5-diphenyltetrazolium bromide (MTT; Sigma-Aldrich) assay. Briefly, cells were seeded in 96-well plates and treated with VPA (0-3.2 $\mathrm{mM})$, alone or in combination with $60 \mu \mathrm{M} 10058-\mathrm{F} 4$, for $24 \mathrm{~h}$. In each well, MTT solution (final concentration, $0.5 \mathrm{mg} / \mathrm{ml}$ ) was added and the cells were then incubated at $37^{\circ} \mathrm{C}$ for $4 \mathrm{~h}$. The absorbance value of each well was measured by a spectrophotometry at $570 \mathrm{~nm}$.

Flow cytometry. Cell death was detected by Annexin V-fluorescein isothiocyanate (FITC) and propidium iodide (PI) (BD Pharmingen, San Diego, CA, USA) staining. Cells were treated with VPA $(0-2.4 \mathrm{mM})$, with or without $60 \mu \mathrm{M} 10058-\mathrm{F} 4$, for $24 \mathrm{~h}$. Cells were collected in a tube using pipet tips, washed twice in $4^{\circ} \mathrm{C}$ PBS and then resuspended in $50 \mu \mathrm{l}$ Annexin $\mathrm{V}$ binding buffer (BD Pharmingen). Subsequently, $5 \mu \mathrm{l}$ Annexin V-FITC and $5 \mu 1 \mathrm{PI}$ were added, and these samples were incubated in the dark at $25^{\circ} \mathrm{C}$ for $15 \mathrm{~min}$. Following the addition of $450 \mu \mathrm{l}$ Annexin V binding buffer, cell death in the samples was measured on a FACScanto ${ }^{\mathrm{TM}}$ II flow cytometer (Becton Dickinson, Franklin Lakes, NJ, USA).

Quantitative polymerase chain reaction ( $q P C R)$. The expression level of the c-MYC gene was determined using reverse transcription-qPCR with SYBR Green I[Takara Biotechnology (Dalian) Co., Ltd., Dalian, China], and GAPDH was used as a reference gene. Total RNA was extracted using TRIzol (Invitrogen Life Technologies, Carlsbad, CA, USA) and reverse transcribed into cDNA by a SuperScript II first-strand cDNA synthesis kit (Invitrogen Life Technologies). The reaction system contained $12.5 \mu 12 \mathrm{X}$ SYBR Premix Ex Taq [Takara Biotechnology (Dalian) Co., Ltd.], $1 \mu 1 \mathrm{cDNAs}$ and 10 pmol of each primer. The primer sequences were as follows: Forward, 5'-ATGGGGAAGGTGAAGGTCG-3' and reverse, 5'-GGGTCATTGATGGCAACAATATC-3' for GAPDH; and forward, 5'-CGTCTCCACACATCAGCACAA-3' and reverse, 5'-CACTGTCCAACTTGACCCTCTTG-3' for c-MYC. The reaction was performed at $95^{\circ} \mathrm{C}$ for $1 \mathrm{~min}$, followed by 40 cycles of denaturation at $95^{\circ} \mathrm{C}$ for $15 \mathrm{sec}$ and annealing/extension at $60^{\circ} \mathrm{C}$ for $60 \mathrm{sec}$, on an iQ5 Real-Time PCR instrument (Bio-Rad Laboratories, Inc., Hercules, CA, USA).

Western blot. Jurkat and CCRF-CEM cells were lysed at $0^{\circ} \mathrm{C}$ in lysis buffer for $30 \mathrm{~min}$, and their protein concentrations were measured by the Bradford protein assay method. The samples were separated by $12 \%$ sodium dodecyl sulfatepolyacrylamide gel electrophoresis and then transferred onto polyvinylidene fluoride (PVDF) membranes. PVDF membranes were blocked with Tris-buffered saline containing $0.1 \%$ Tween (TBST; prepared in the laboratory, Department of Hematology, Institute of Hematology, The First Affiliated Hospital, Zhejiang University School of Medicine, Hangzhou, China) and 5\% non-fat milk for $2 \mathrm{~h}$ at room temperature. The membranes were then incubated with the primary antibodies, rabbit anti-human monoclonal c-Myc (D84C12 $\left.\mathrm{XP}^{\circledR}\right)$, rabbit anti-human monoclonal caspase-3 (8G10) and mouse antihuman monoclonal $\beta$-actin (8H10D10) (dilution, 1:1000-5000; Cell Signaling Technology, Beverly, MA, USA), overnight at $4^{\circ} \mathrm{C}$. Following washing four times for $10 \mathrm{~min}$ each time with TBST, these PVDF membranes were incubated with the goat anti-rabbit (catalogue number, 7074) or horse anti-mouse (catalogue number, 7076) IgG horseradish peroxidase-conjugated secondary antibodies (dilution, 1:2000; Cell Signaling Technology) for $2 \mathrm{~h}$ at room temperature. The membranes were again washed four times with TBST, and protein bands were then visualized with the enhanced chemiluminescence detecting kit (EZ-ECL chemiluminescence detection kit; Biological Industries, Beit-Haemek, Israel) and exposed to X-ray films.

Statistical analysis. The significant differences between experimental and control groups were compared by Student's t-test. $\mathrm{P}<0.05$ was considered to indicate a statistically significant difference. All statistical analyses were performed using SPSS 16.0 software (SPSS Inc., Chicago, IL, USA).

\section{Results}

VPA downregulates the expression of c-Myc in Jurkat and CCRF-CEM cells. First, the role of c-Myc in the growth inhibition of T-ALL cells induced by VPA was identified. Western blots revealed that after Jurkat and CCRF-CEM cells were treated with VPA at various concentrations for $24 \mathrm{~h}$, the expression of c-Myc protein was markedly downregulated in a dose-dependent manner, and was accompanied by cleavage of caspase-3 (Fig. 1A). qPCR was then performed to measure the c-Myc mRNA levels, and it was found that the expression of c-Myc was also decreased in Jurkat and CCRF-CEM cells treated with $0.8 \mathrm{mM}$ VPA for $24 \mathrm{~h}$ (Fig. 1B; P<0.01), indicating that $\mathrm{c}-\mathrm{Myc}$ was downregulated at a transcriptional level. In addition, decreased expression of c-Myc protein in a time-dependent manner was observed in Jurkat cells (Fig. 1C), but not examined in CCRF-CEM cells.

10058-F4 further promotes downregulation of c-Myc expression by VPA. It has been demonstrated that 10058-F4 can block the dimerization of c-Myc and Max, and then inhibit c-Myc transactivating activity (15). Thus, we hypothesized that 10058-F4 promotes the downregulation of c-Myc expression induced by VPA. The western blotting results showed that c-Myc expression levels in Jurkat and CCRF-CEM cells treated with VPA $(0,0.8$ and $1.6 \mathrm{mM}$ ) combined with $60 \mu \mathrm{M} 10058-\mathrm{F} 4$ decreased further compared with the corresponding controls (Fig. 2).

10058-F4 increases the growth inhibition of Jurkat and CCRF-CEM cells induced by VPA. c-Myc is an important 
A

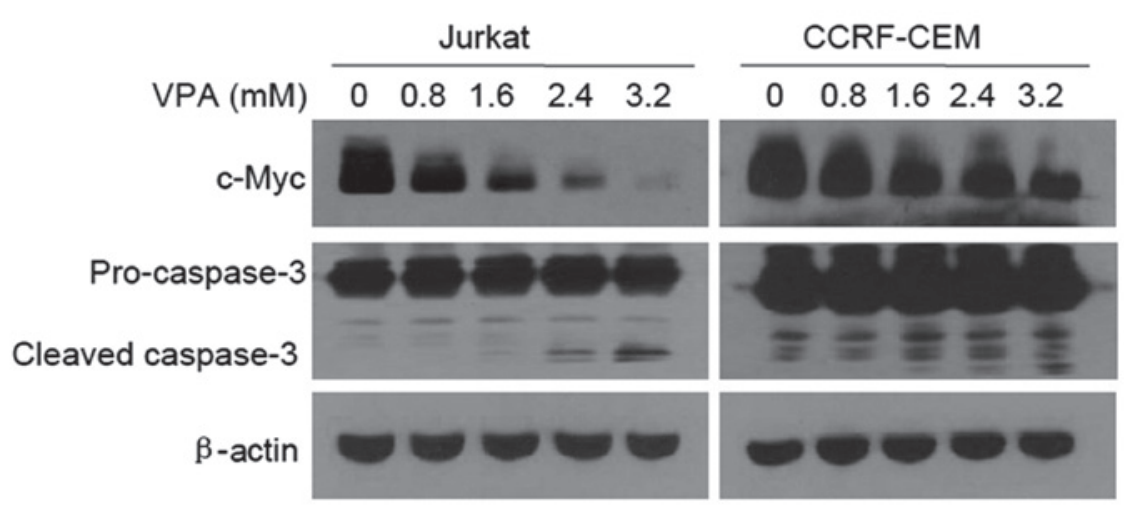

B

c-Myc mRNA expression

C

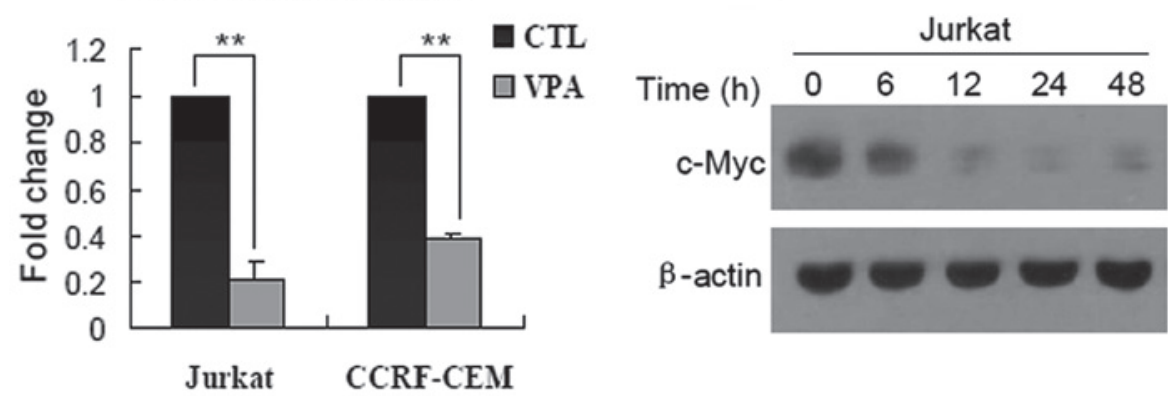

Figure 1. Influence of VPA on the expression levels of c-Myc. (A) Level of c-Myc protein varied in Jurkat and CCRF-CEM cells exposed to 0.8, 1.6, 2.4 and $3.2 \mathrm{mM}$ VPA. (B) Level of c-Myc mRNA varied in Jurkat and CCRF-CEM cells exposed to $0.8 \mathrm{mM}$ VPA. (C) Level of c-Myc protein varied in Jurkat cells exposed to $0.8 \mathrm{mM}$ VPA for $6,12,24$ and $48 \mathrm{~h}$. ${ }^{* * *} \mathrm{P}<0.01$. VPA, valproic acid.

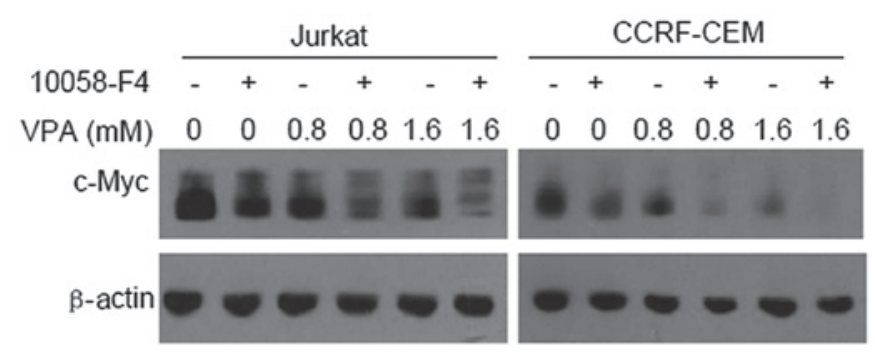

Figure 2. Levels of c-Myc protein in Jurkat and CCRF-CEM cells exposed to VPA combined with 10058-F4 for 24 h. VPA, valproic acid.

oncogene, which contributes to the growth of T-ALL cells, particularly T-ALL cells with Notch1 mutations (5). The present study results showed that 10058-F4 and VPA synergistically downregulated c-Myc expression in Jurkat and CCRF-CEM cells. It was next investigated whether 10058-F4 could increase cell growth inhibition induced by VPA in Jurkat and CCRF-CEM cells, by MTT assay. The growth inhibition rates of Jurkat cells treated with VPA $(0,0.8,1.6$ and $2.4 \mathrm{mM}$ ) combined with $10058-\mathrm{F} 4$ for $24 \mathrm{~h}$ were $17.06 \pm 1.03$, $47.99 \pm 4.35,51.70 \pm 6.19$ and $65.27 \pm 6.86 \%$ respectively, which were significantly higher than those treated with corresponding concentrations of VPA $(0,4.28 \pm 0.13,11.21 \pm 4.46$ and $17.86 \pm 2.60 \%)(\mathrm{P}=0.012)$. In CCRF-CEM cells, the growth inhibition rates for VPA $(0,0.8,1.6$ and $2.4 \mathrm{mM})$ plus $10058-\mathrm{F} 4$ were $23.80 \pm 3.37,55.76 \pm 3.72,64.65 \pm 2.48$ and $68.60 \pm 3.48 \%$, respectively, which were also significantly higher than those for the corresponding concentrations of VPA alone $(0,7.92 \pm 2.62$, $17.03 \pm 4.54$ and $17.42 \pm 3.42 \%)(\mathrm{P}=0.007)($ Fig. 3).
10058-F4 markedly increases the cell death induced by VPA in a caspase-dependent and-independent manner. Previous studies have demonstrated that 10058-F4 efficiently induced cell death in myeloma and AML cells $(15,16)$. Concordant with this, cell death of Jurkat and CCRF-CEM cells treated with VPA $(0,0.8,1.6$ and $2.4 \mathrm{mM})$ was increased when combined with 10058-F4. As shown in Fig. 4A, compared with treatment with corresponding concentrations of VPA alone, cell death rates (Annexin $\mathrm{V}^{+} / \mathrm{PI}^{+}$and Annexin $\mathrm{V}^{+} / \mathrm{PI}^{-}$) of Jurkat and CCRF-CEM cells treated with 10058-F4 combined with VPA increased significantly ( $\mathrm{P}=0.038$ and $\mathrm{P}=0.037$, respectively). In addition, western blot analysis revealed that 10058-F4 could promote the cleavage of caspase-3 induced by VPA (Fig. 4B). The results also demonstrated thatZ-VAD-FMK, a pan-caspase inhibitor, could partially inhibit cell death of Jurkat cells induced by $10058-\mathrm{F} 4$ combined with VPA $(72.33 \pm 3.35$ vs. $37.4 \pm 1.87 \%, \mathrm{P}<0.001$ ) (Fig. $4 \mathrm{C}$ ). These findings indicate that 10058-F4 dramatically increases cell death induced by VPA through caspase-dependent and -independent pathways.

\section{Discussion}

It has been demonstrated that VPA could prevent growth of cancer cells by inducing apoptosis and cell cycle arrest, and by promoting cellular differentiation (17). HDACIs, including VPA, can selectively alter the expression levels of a relatively small proportion of genes by recovering the deacetylation of histones (18). Regarding these genes in which the expression levels are altered by HDACIs, upregulation of the surface TRAIL death receptors (DR4 and DR5) in multiple myeloma (19), upregulation of p21 and p27 in mantle cell 

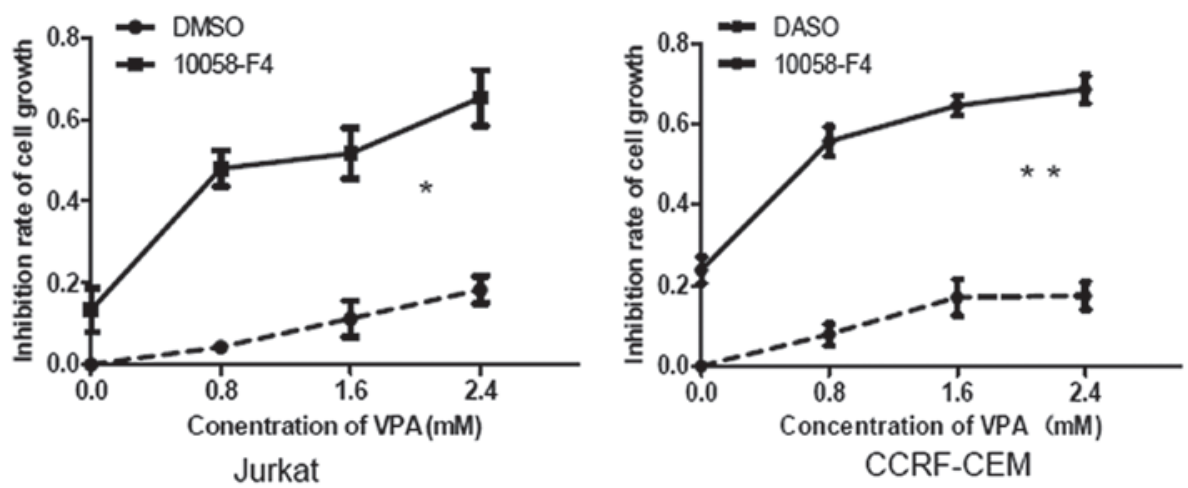

Figure 3. Growth inhibition rates of Jurkat and CCRF-CEM cells exposed to VPA combined with 10058-F4 (60 $\mu \mathrm{M})$ for $24 \mathrm{~h}$. ${ }^{*} \mathrm{P}<0.05$ and ${ }^{* *} \mathrm{P}<0.01$ for all concentrations of VPA. VPA, valproic acid.

A
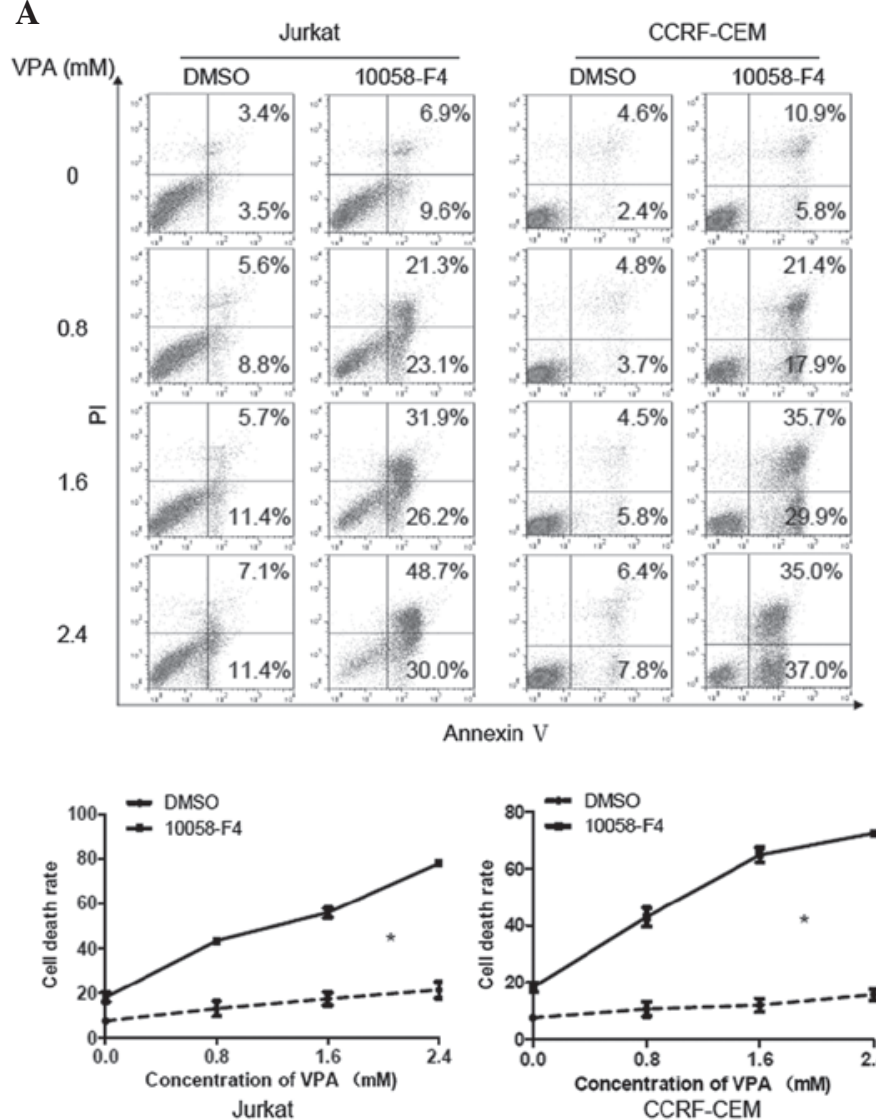

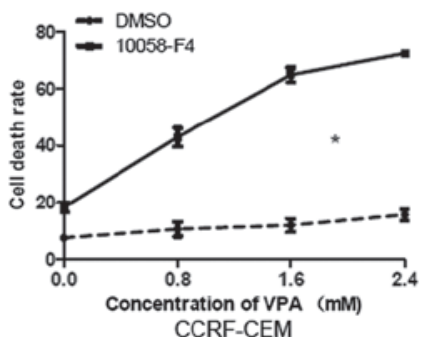

CCRF-CEM
B

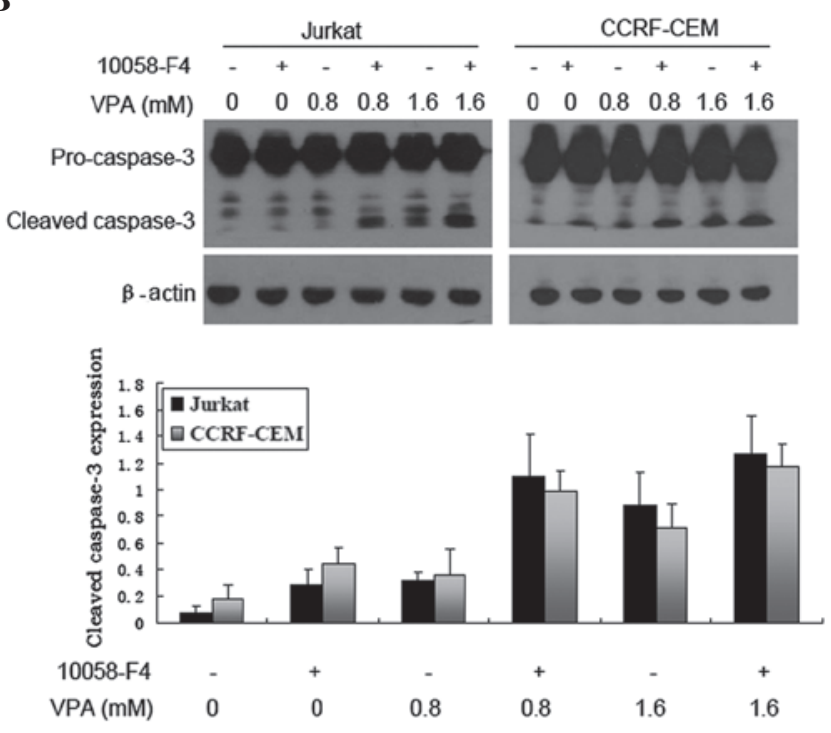

C

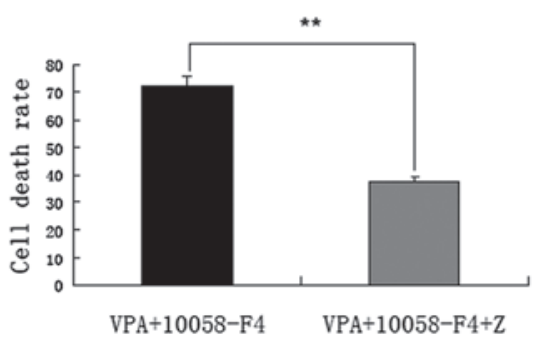

Figure 4. Cell death of Jurkat and CCRF-CEM cells induced by VPA combined with 10058-F4. (A) Cell death was examined by dual staining with Annexin V/PI in Jurkat and CCRF-CEM cells treated with VPA combined with 10058-F4 (60 $\mu \mathrm{M})$ for $24 \mathrm{~h}$ (mean $\pm \mathrm{SD}$; $\mathrm{n}=3)$. (B) The level of cleaved caspase-3 varied after exposure of cells to VPA combined with $60 \mu \mathrm{M} 10058$-F4. Bar graphs were plotted according to the densitometry of the cleaved caspase-3/ $\beta$-actin band densities (mean $\pm \mathrm{SD} ; \mathrm{n}=3$ ). (C) Cell death induced by VPA $(2.4 \mathrm{mM})$ combined with 10058-F4 (60 $\mu \mathrm{M})$ in Jurkat cells was partially inhibited by Z-VAD-FMK $(20 \mu \mathrm{M})($ mean $\pm \mathrm{SD} ; \mathrm{n}=3)$. *" $\mathrm{P}<0.01$ for all concentrations of VPA. VPA, valproic acid; PI, propidium iodide; Z, Z-VAD-FMK.

lymphoma (20), and downregulation of c-Myc in AML and endometrial cancer cells $(13,21)$ played a key role in the induction of apoptosis and cell cycle arrest or the promotion of cellular differentiation.

Since activating mutations of Notch1 were observed in $>50 \%$ T-ALL patients (3) and c-Myc is an important direct target of Notch1 (5), the present study examined whether c-Myc was downregulated in the growth inhibition of T-ALL cells induced by VPA. The results showed that, as in AML (13),
c-Myc expression was markedly downregulated in Jurkat and CCRF-CEM cells in which activation of Notch1 signaling occurred (22). Further investigation revealed that 10058-F4 could reinforce the downregulation of c-Myc induced by VPA, indicating that the former may increase the sensitivity to the latter.

Previous studies have demonstrated that VPA can induce apoptosis in leukemic cells $(7,23)$. Notably, VPA also augmented apoptosis of leukemic cells induced by other agents, such as 
cytarabine (24), etoposide (25) and bortezomib (26). Thus, the present study next examined the cell death of Jurkat and CCRF-CEM cells induced by VPA combined with 10058-F4. The results showed that 10058-F4 could markedly increase the cell death of Jurkat and CCRF-CEM cells induced by VPA $(0,0.8,1.6$ and $2.4 \mathrm{mM})$. Activation of caspase-3 also increased correspondingly. However, Z-VAD-FMK partially inhibited the cell death induced by 10058-F4 combined with VPA, indicating that their apoptotic effects involved in both caspase-dependent and -independent pathways. Previous studies have also demonstrated that not only 10058-F4, but also VPA, induced apoptosis through both caspase-dependent and -independent pathways $(15,23,27)$.

Although there have been arguments for and against the therapeutic targeting of Myc (28), recent experiments in vivo and in vitro have demonstrated that it is a promising therapeutic strategy in high-risk hematologic malignancies $(29,30)$. The present study showed that downregulation of c-Myc by 10058-F4 markedly increased VPA-induced apoptosis. These findings suggest that VPA combined with c-Myc inhibitors may be a novel potent therapeutic strategy for adult T-ALL patients. However, further investigation with regard to the clinical effect of their combination is required in the future.

\section{Acknowledgements}

This study was supported by the Foundation of Innovation Team for Basic and Clinical Research of Zhejiang Province (grant no. 2011R50015), the National Public Health Grand Research Foundation (grant no. 201202017) and the Foundation of Ningbo Medical Science and Technology Project (grant no. 2011A02).

\section{References}

1. Demarest RM, Ratti F and Capobianco AJ: It's T-ALL about Notch. Oncogene 27: 5082-5091, 2008.

2. Weng AP, Ferrando AA, Lee W, et al: Activating mutations of NOTCH1 in human $\mathrm{T}$ cell acute lymphoblastic leukemia. Science 306: 269-271, 2004.

3. Sarmento LM and Barata JT: Therapeutic potential of Notch inhibition in T-cell acute lymphoblastic leukemia: rationale, caveats and promises. Expert Rev Anticancer Ther 11: 1403-1415, 2011.

4. Thompson BJ, Jankovic V, Gao J, et al: Control of hematopoietic stem cell quiescence by the E3 ubiquitin ligase Fbw7. J Exp Med 205: 1395-1408, 2008.

5. Weng AP, Millholland JM, Yashiro-Ohtani Y, et al: c-Myc is an important direct target of Notch1 in T-cell acute lymphoblastic leukemia/lymphoma. Genes Dev 20: 2096-2109, 2006.

6. Malempati S, Tibbitts D, Cunningham M, et al: Aberrant stabilization of c-Myc protein in some lymphoblastic leukemias. Leukemia 20: 1572-1581, 2006.

7. Shao N, Ma D, Wang J, Lu T, Guo Y and Ji C: Notch1 signaling is irresponsible to the anti-leukemic effect of HDACis in B-ALL Nalm-6 cells. Ann Hematol 92: 33-39, 2013.

8. Nie D, Huang K, Yin S, et al: Synergistic/additive interaction of valproic acid with bortezomib on proliferation and apoptosis of acute myeloid leukemia cells. Leuk Lymphoma 53: 2487-2495, 2012.

9. Kuendgen A, Knipp S, Fox F, et al: Results of a phase 2 study of valproic acid alone or in combination with all-trans retinoic acid in 75 patients with myelodysplastic syndrome and relapsed or refractory acute myeloid leukemia. Ann Hematol 84: 61-66, 2005.
10. Sanchez-Gonzalez B, Yang H, Bueso-Ramos C, et al: Antileukemia activity of the combination of an anthracycline with a histone deacetylase inhibitor. Blood 108: 1174-1182, 2006.

11. Yang H, Hoshino K, Sanchez-Gonzalez B, Kantarjian H and Garcia-Manero G: Antileukemia activity of the combination of 5-aza-2'-deoxycytidine with valproic acid. Leuk Res 29: $739-748,2005$

12. Leiva M, Moretti S, Soilihi H, et al: Valproic acid induces differentiation and transient tumor regression, but spares leukemia-initiating activity in mouse models of APL. Leukemia 26: 1630-1637, 2012.

13. Cheng YC, Lin H, Huang MJ, Chow JM, Lin S and Liu HE: Downregulation of c-Myc is critical for valproic acid-induced growth arrest and myeloid differentiation of acute myeloid leukemia. Leuk Res 31: 1403-1411, 2007.

14. Kutuk O, Pedrech A, Harrison P and Basaga H: Pramanicin induces apoptosis in Jurkat leukemia cells: a role for JNK, p38 and caspase activation. Apoptosis 10: 597-609, 2005.

15. Huang MJ, Cheng YC, Liu CR, Lin S and Liu HE: A small-molecule c-Myc inhibitor, 10058-F4, induces cell-cycle arrest, apoptosis, and myeloid differentiation of human acute myeloid leukemia. Exp Hematol 34: 1480-1489, 2006.

16. Holien T, Vatsveen TK, Hella H, et al: Bone morphogenetic proteins induce apoptosis in multiple myeloma cells by Smad-dependent repression of MYC. Leukemia 26: 1073-1080, 2012.

17. Masetti R, Serravalle S, Biagi C and Pession A: The role of HDACs inhibitors in childhood and adolescence acute leukemias. J Biomed Biotechnol 2011: 148046, 2011.

18. Dokmanovic M, Clarke C and Marks PA: Histone deacetylase inhibitors: overview and perspectives. Mol Cancer Res 5: 981-989, 2007

19. Fandy TE, Shankar S, Ross DD, Sausville E and Srivastava RK: Interactive effects of HDAC inhibitors and TRAIL on apoptosis are associated with changes in mitochondrial functions and expressions of cell cycle regulatory genes in multiple myeloma. Neoplasia 7: 646-657, 2005.

20. Heider U, Kaiser M, Sterz J, et al: Histone deacetylase inhibitors reduce VEGF production and induce growth suppression and apoptosis in human mantle cell lymphoma. Eur J Haematol 76: 42-50, 2006.

21. Zhao ZN, Bai JX, Zhou Q, et al: TSA suppresses miR-106b-93-25 cluster expression through downregulation of MYC and inhibits proliferation and induces apoptosis in human EMC. PLoS One 7: e45133, 2012.

22. O'Neil J, Grim J, Strack P, et al: FBW7 mutations in leukemic cells mediate NOTCH pathway activation and resistance to gamma-secretase inhibitors. J Exp Med 204: 1813-1824, 2007.

23. Kawagoe R, Kawagoe H and Sano K: Valproic acid induces apoptosis in human leukemia cells by stimulating both caspase-dependent and -independent apoptotic signaling pathways. Leuk Res 26: 495-502, 2002.

24. Xie C, Edwards H, Xu X, et al: Mechanisms of synergistic antileukemic interactions between valproic acid and cytarabine in pediatric acute myeloid leukemia. Clin Cancer Res 16: 5499-5510, 2010.

25. Jasek E, Lis GJ, Jasinska M, Jurkowska $H$ and Litwin JA: Effect of histone deacetylase inhibitors trichostatin A and valproic acid on etoposide-induced apoptosis in leukemia cells. Anticancer Res 32: 2791-2799, 2012.

26. Wang AH, Wei L, Chen L, et al: Synergistic effect of bortezomib and valproic acid treatment on the proliferation and apoptosis of acute myeloid leukemia and myelodysplastic syndrome cells. Ann Hematol 90: 917-931, 2011.

27. Schwartz C, Palissot V, Aouali N, et al: Valproic acid induces non-apoptotic cell death mechanisms in multiple myeloma cell lines. Int J Oncol 30: 573-582, 2007.

28. Prochownik EV and Vogt PK: Therapeutic Targeting of Myc. Genes Cancer 1: 650-659, 2010.

29. Ott CJ, Kopp N, Bird L, et al: BET bromodomain inhibition targets both c-Myc and IL7R in high-risk acute lymphoblastic leukemia. Blood 120: 2843-2852, 2012.

30. Filippakopoulos P, Qi J, Picaud S, et al: Selective inhibition of BET bromodomains. Nature 468: 1067-1073, 2010. 